\title{
Research Article Substrate Integrated Waveguide Cross-Coupling Filter with Multilayer Hexagonal Cavity
}

\author{
B. Wu, Z. Q. Xu, and J. X. Liao \\ Research Institute of Electronic Science and Technology, University of Electronic Science and Technology of China, \\ Chengdu 611731, China \\ Correspondence should be addressed to Z. Q. Xu; nanterxu@uestc.edu.cn
}

Received 27 August 2013; Accepted 12 October 2013

Academic Editors: J. Charrier, V. Laude, V. M. N. Passaro, and J.-K. Xiao

Copyright (C) 2013 B. Wu et al. This is an open access article distributed under the Creative Commons Attribution License, which permits unrestricted use, distribution, and reproduction in any medium, provided the original work is properly cited.

Hexagonal cavities and their applications to multilayer substrate integrated waveguide (SIW) filters are presented. The hexagonal SIW cavity which can combine flexibility of rectangular one and performance of circular one is convenient for bandpass filter's design. Three types of experimental configuration with the same central frequency of $10 \mathrm{GHz}$ and bandwidth of $6 \%$, including three-order and four-order cross-coupling topologies, are constructed and fabricated based on low temperature cofired ceramic (LTCC) technology. Both theoretical and experimental results are presented.

\section{Introduction}

Waveguide filters are widely used in various microwave and millimeter-wave communication systems, especially airborne platforms, communication satellites, earth stations, and wireless base stations, due to their high quality factor $(Q-$ factor) and high power capability. However, they are bulky and not suitable for high-density integration, which greatly increases the cost of the entire system $[1,2]$. Recently substrate integrated waveguide (SIW) which is synthesized in a planar substrate with arrays of metallic via provides a low-profile, low-weight, and low-cost solution while maintaining high performance $[3,4]$.

Recently, various types of SIW filters have been paid much attention. It is known that conventional structures of SIW filters are predominantly based on rectangular and circular cavities $[5,6]$. Rectangular cavity is superior for its flexible structure while circular cavity is for its high performance [7-9]. To combine their predominance together, novel SIW filters with hexagonal resonators are proposed in [10]. Since any of the six sides of a hexagonal cavity can be utilized for coupling, it is flexible in the design of SIW filter. However, their physical sizes are not compact enough due to their planar and single-layer structure. Besides, it is usually not easy to implement negative coupling in planar structure owing to similar coupling manner between the adjacent resonators in the same plane.

In this paper, in regard to compact size and stringent frequency selectivity, multilayer hexagonal cavities which combine flexibility of rectangular cavities and performance of circular cavities and their applications to SIW filters are proposed. Three types of experimental filter configuration at the same central frequency of $10 \mathrm{GHz}$ are fabricated using low temperature cofired ceramic (LTCC) technology. Design details are described, and both simulated and experimental results are presented and discussed.

\section{Filter Analysis and Design}

2.1. Hexagonal Resonant Cavity. The configurations and electric field distributions of the fundamental mode of a hexagonal cavity resonator are shown in Figure 1. Its electric field distributions and resonant characteristics are similar with circular resonators.

Here, $L, a$, and $p$ stand for length of regular hexagonal cavity, diameter of metallized via holes, and pitch between them, respectively. There is no accurate formula between 
TABLE 1: Design goal of filters A, B, and C.

\begin{tabular}{lcccc}
\hline Filter types & Center frequency & Fractional bandwidth & Return loss & Transmission zeros \\
\hline Filter A & $10 \mathrm{GHz}$ & $6 \%$ & $20 \mathrm{~dB}$ & $10.3 \mathrm{GHz}$ \\
Filter B & $10 \mathrm{GHz}$ & $6 \%$ & $20 \mathrm{~dB}$ & $9.6 \mathrm{GHz}$ \\
Filter C & $10 \mathrm{GHz}$ & $6 \%$ & $20 \mathrm{~dB}$ & $9.6,10.5 \mathrm{GHz}$ \\
\hline
\end{tabular}

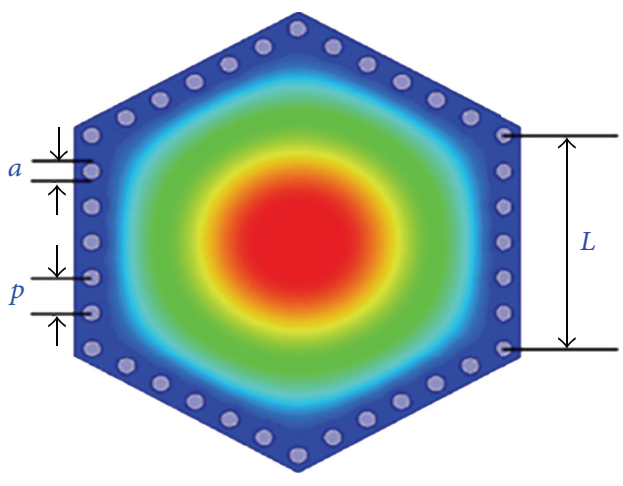

FIGURE 1: The configurations and electric field distributions of the fundamental mode of a hexagonal cavity resonator.

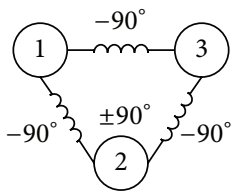

Filter A

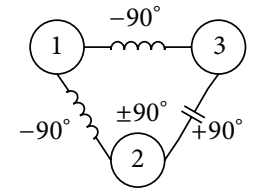

Filter B

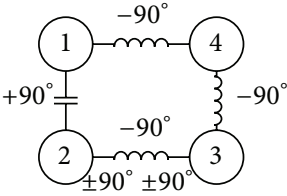

Filter C
Figure 2: Coupling topological structures of Filters A, B, and C.

geometrical parameters and eigenfrequencies of a hexagonal resonator yet, but [10] proposed an approximate expression as

$$
L=\frac{c}{\sqrt{\varepsilon_{r}}} \cdot \frac{\mu^{\prime}}{2 \pi f_{r}} .
$$

Here, $c, \varepsilon_{r}$, and $f_{r}$ stand for speed of light, relative permittivity of dielectric substrate and eigen frequencies of SIW hexagonal resonator, respectively. $\mu^{\prime}=2.75$ is the modified root coefficient based on Bessel function.

2.2. Analysis of the Proposed Filters. Taking three-order and four-order filters as examples, three types of novel multilayer SIW hexagonal cavity filters are proposed, with design goals illustrated in Table 1.

Abstracted coupling coefficients determine coupling patterns and initial geometrical dimensions, while calculating normalized frequency of prototype lowpass filter in advance as

$$
\Omega=\frac{\omega_{0}}{\Delta \omega}\left(\frac{\omega}{\omega_{0}}-\frac{\omega_{0}}{\omega}\right) .
$$

For Filters A and C, their coupling matrixes have negative elements on secondary diagonal, unlike direct coupling. Magnetic coupling pattern contributes to achieve crosscoupling in multilayer structure. For Filter $A, S_{11}$ remains and $S_{21}$ inverses while inversing $M_{13}, M_{31}, M_{12}$, and $M_{21}$ simultaneously. For Filter $\mathrm{C}, \mathrm{S}_{11}$ remains and $\mathrm{S}_{21}$ inverses while inversing $\mathrm{M}_{41}, \mathrm{M}_{12}, \mathrm{M}_{21}$, and $\mathrm{M}_{21}$ simultaneously. Transformed coupling matrixes and their $Q$-factors are shown as follows:

$$
\begin{gathered}
M_{\mathrm{A}}=\left[\begin{array}{ccc}
0.0082 & 0.0546 & 0.0341 \\
0.0546 & -0.0325 & 0.0546 \\
0.0341 & 0.0546 & 0.0082
\end{array}\right], \quad \mathrm{Q}_{\mathrm{A}}=15.3857, \\
M_{\mathrm{B}}=\left[\begin{array}{ccc}
-0.0082 & 0.0546 & 0.0341 \\
0.0546 & 0.0325 & -0.0546 \\
0.0341 & -0.0546 & -0.0082
\end{array}\right], \quad \mathrm{Q}_{\mathrm{B}}=15.3857, \\
M_{\mathrm{C}}=\left[\begin{array}{cccc}
0 & -0.0435 & 0 & 0.0085 \\
-0.0435 & 0 & 0.0384 & 0 \\
0 & 0.0384 & 0 & 0.0435 \\
0.0085 & 0 & 0.0435 & 0
\end{array}\right], \\
\mathrm{Q}_{\mathrm{C}}=19.5396 .
\end{gathered}
$$

Normally, coupling coefficient is positively correlated with size of coupling window, which can be preliminarily determined by their relational curve graph.

We define negative coupling coefficient as electric coupling and positive coupling coefficient as magnetic coupling. Numbered circle stands for each resonator, and the symbols of inductance and capacitance represent the opposite coupling relationships between two connective resonators, respectively. Hence, coupling topological structures of Filters A, B and C are shown in Figure 2.

For Filter A, signal passed primary channel obtains opposite phase against that passed cross-coupling channel and forms a transmission zero beyond passband. Similarly, Filter B forms a transmission zero underneath passband, while Filter $\mathrm{C}$ forms two at both outsides of pass band.

Magnetic coupling is achieved by slotting at the area with maximum magnetic field intensity on common surface between cavities, while electric coupling with maximum electric field intensity. 


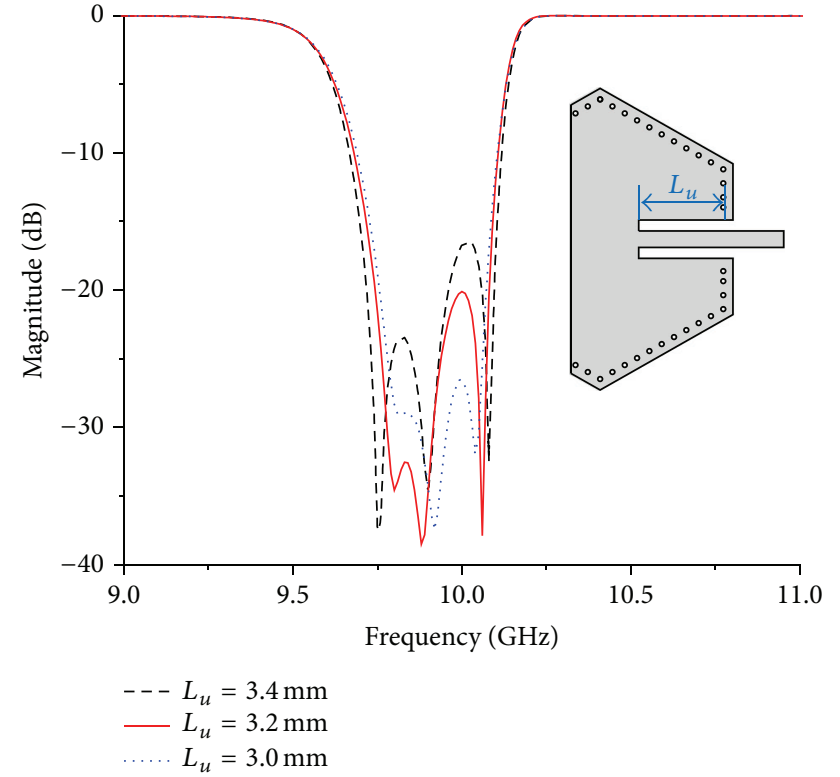

Figure 3: Effect of GCPW's length on return loss in Filter A.

TABLE 2: Comparisons with some conventional SIW filters.

\begin{tabular}{lccc}
\hline References & $f_{\text {center }}$ & BW & Occupying sizes \\
\hline$[10]$ & $10 \mathrm{GHz}$ & $6 \%$ & $>30 \times 30 \mathrm{~mm}^{2}$ \\
{$[11]$} & $10 \mathrm{GHz}$ & $3.3 \%$ & $>30 \times 30 \mathrm{~mm}^{2}$ \\
{$[12]$} & $6 \mathrm{GHz}$ & $5 \%$ & $>48 \times 24 \mathrm{~mm}^{2}$ \\
{$[13]$} & $10 \mathrm{GHz}$ & $3.4 \%$ & $>32 \times 32 \mathrm{~mm}^{2}$ \\
This work & $10 \mathrm{GHz}$ & $6 \%$ & $22 \times 12 \mathrm{~mm}^{2}$ \\
\hline
\end{tabular}

2.3. Parameters of the Proposed Filters. External Q-factors determined by input and output structures play a crucial role in suppressing return loss within pass band. Input and output adopt electric current probe form, which consist of GCPW and $50 \Omega$ microstrip line. Width of GCPW's gap is assigned as $0.25 \mathrm{~mm}$ for preventing power leakage and machining tolerance.

Effect of GCPW's length on return loss in Filter A is shown in Figure 3. Loaded $Q$-factor varies due to length's change, causing variation of return loss within pass band. Hence, it is important for ports' dimensions to return loss within pass band.

Transmission zero comes from cross-coupling when signal passed primary channel obtains $180^{\circ}$ phase shift against that passed cross-coupling channel at corresponding frequency point. Therefore, cross-coupling has a great impact on transmission zero. Effect of coupling window's length on transmission zero in Filter B is shown in Figure 4. Coupling intensity between two resonant cavities is enhanced as length elongating, causing transmission zero underneath pass band to move downward.

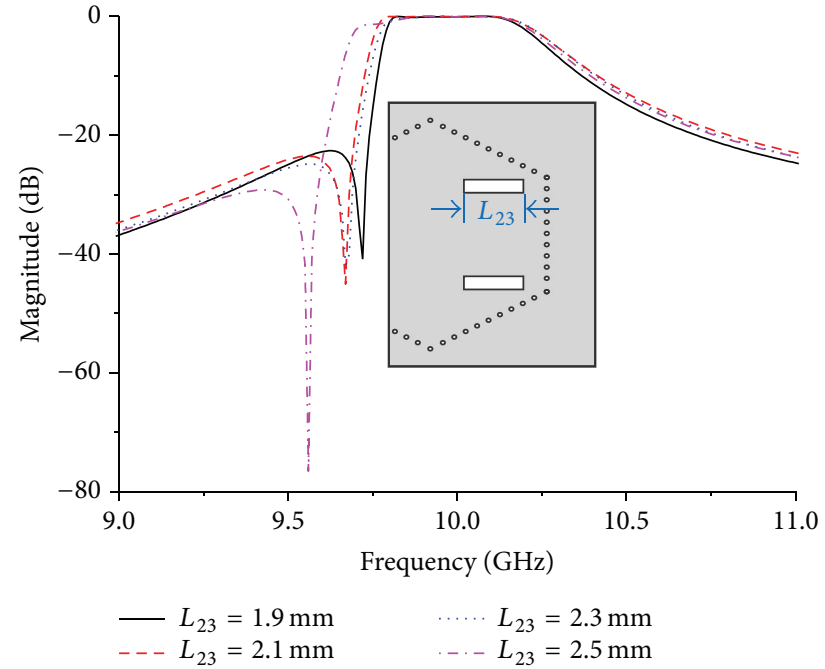

FIGURE 4: Effect of coupling window's length on transmission zero in filter B.

After theory analysis, simulation and optimization, final dimensions and photographs of Filters A, B, and C are shown in Figures 5 and 6, respectively.

\section{Fabrication and Experimental Results}

The proposed three types of filters are implemented on a LTCC substrate with relative permittivity of 5.9, loss tangent of 0.0015 , and thickness of $0.8 \mathrm{~mm}$. Each resonant cavity, up and down surfaces of which being covered with gold, consists of four substrate layers within linear arrays of metallization via holes having diameter of $0.17 \mathrm{~mm}$ and center-to-center pitch of $0.45 \mathrm{~mm}$. Figure 6 shows the photograph of fabricated Filters A, B, and C. The external shapes of fabricated filters are $22 \mathrm{~mm} \times 11.5 \mathrm{~mm} \times 0.8 \mathrm{~mm}, 22 \mathrm{~mm} \times 11.5 \mathrm{~mm} \times$ $0.8 \mathrm{~mm}$, and $22 \mathrm{~mm} \times 12 \mathrm{~mm} \times 0.8 \mathrm{~mm}$, respectively.

An Agilent E8363B vector network analyzer is used for measurement. The simulated results and measured frequency responses are illustrated in Figure 7.

As can be seen, the measured results are in good agreement with the simulated ones. The measured responses of Filters A, B, and C have the fractional bandwidths of 5.83\%, $5.95 \%$, and $5.76 \%$, respectively. The minimum passband insertion loss of Filter A is $1.82 \mathrm{~dB}, 1.68 \mathrm{~dB}$ for Filter B, and that of Filter $\mathrm{C}$ is $1.89 \mathrm{~dB}$. The measured in-band return loss is greater than $16 \mathrm{~dB}$ for Filter $\mathrm{A}, 17 \mathrm{~dB}$ for Filter $\mathrm{B}$, and $16 \mathrm{~dB}$ for Filter C, respectively.

By comparing the measured and simulated results, a small frequency shift and a little discrepancy in the in-band insertion loss are observed. The frequency shift is mainly caused by the difference between the actual and nominal values of the dielectric constant. The degeneration of the in-band insertion loss contributed to the test fixture and the manufacturing tolerance in LTCC process, including dielectric and printed conductor loss, wrinkles of conductor surface and edge, and 


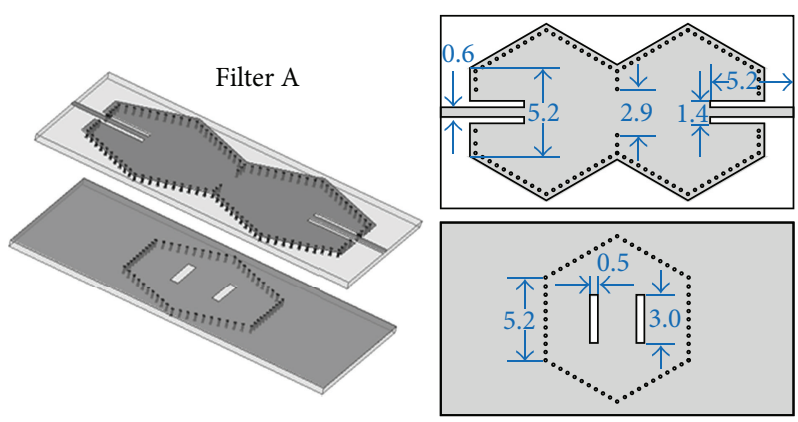

(a)

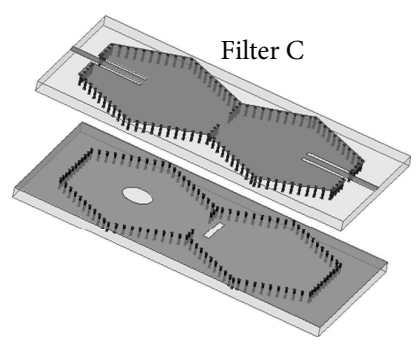

(c)

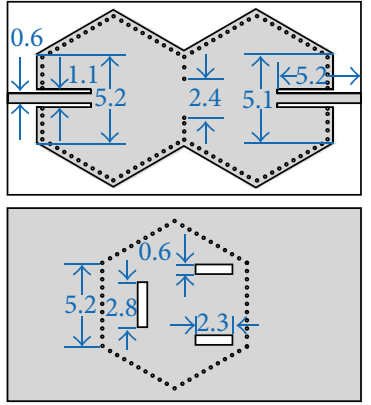

(b)
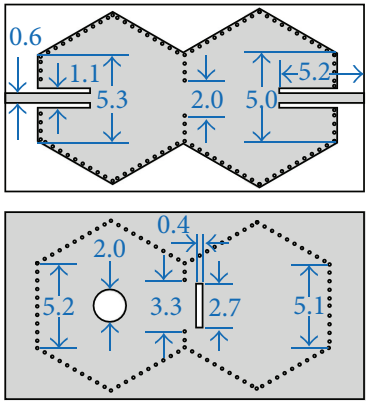

Figure 5: Final dimensions of Filters A, B and C. (unit mm).

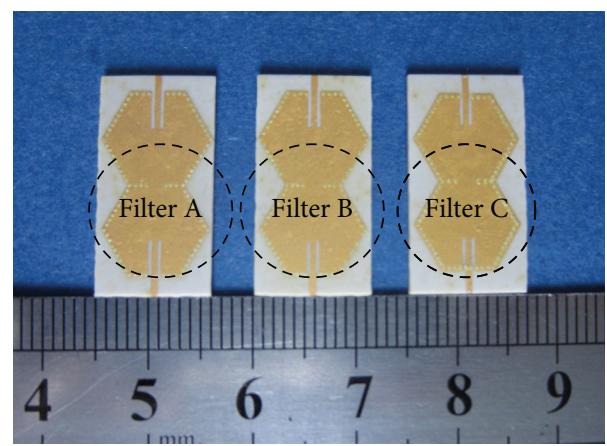

Figure 6: Photographs of fabricated Filters A, B and C.

cofireability of conductor and ceramic material. It should be noted that cofireability between conductor and ceramic during the manufacturing process is the main cause of the degeneration of the in-band insertion loss. If the material or process conditions of the ceramic and conductor are inappropriate, various macro and micro flaws may occur, which will worsen the in-band insertion loss. Meanwhile, in the cofiring process, minute pores may be found forming at the interface, which is yet another possible factor influencing the in-band insertion loss. To avoid the above undesirable possibilities, it is not sufficient only to optimize the cofiring process condition or profile. Rather, it is also necessary in some cases to revise the base powder of each material in order to improve the cofire ability behavior of the conductor and ceramic.

Overall, the proposed filter exhibits good frequency selectivity and compact size by profit from its modified ED and multilayer structure. Table 2 shows the comparison with some other recent works on SIW filters. As can be seen, the proposed filter not only exhibits good selectivity owing to its flexible hexagonal cavities but also has a compact size by profit from multilayer LTCC structure.

\section{Conclusion}

Three types of novel SIW cross-coupling filters with multilayer hexagonal cavity based on LTCC technology are proposed and fabricated. N-2 transmission zeros in proposed n-order filers are achieved by introducing cross-coupling in multilayer substrate. The proposed filters have advantages of low insertion loss and good suppression at outband due to the flexible construction and relative high $Q$-factor of hexagonal resonant cavity, which demonstrate that the 


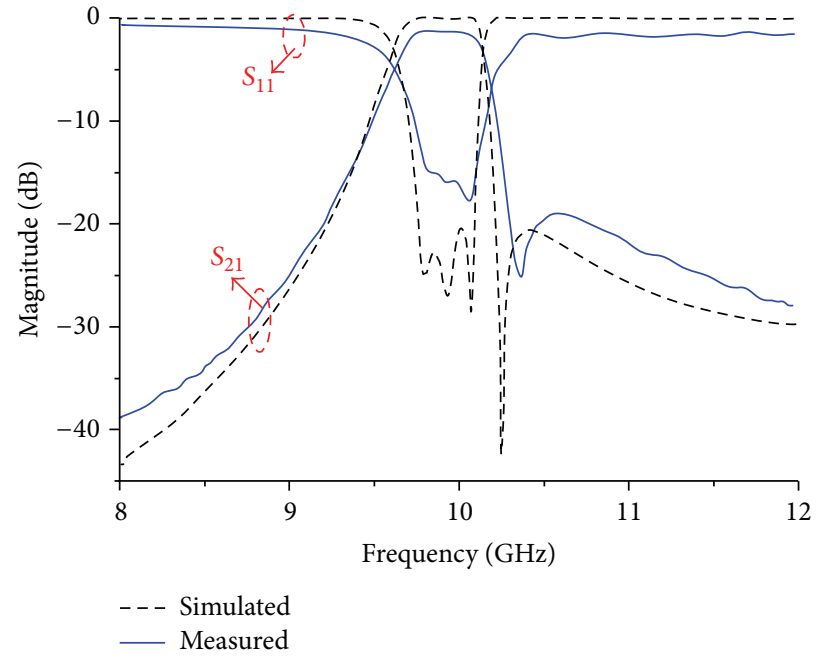

(a)

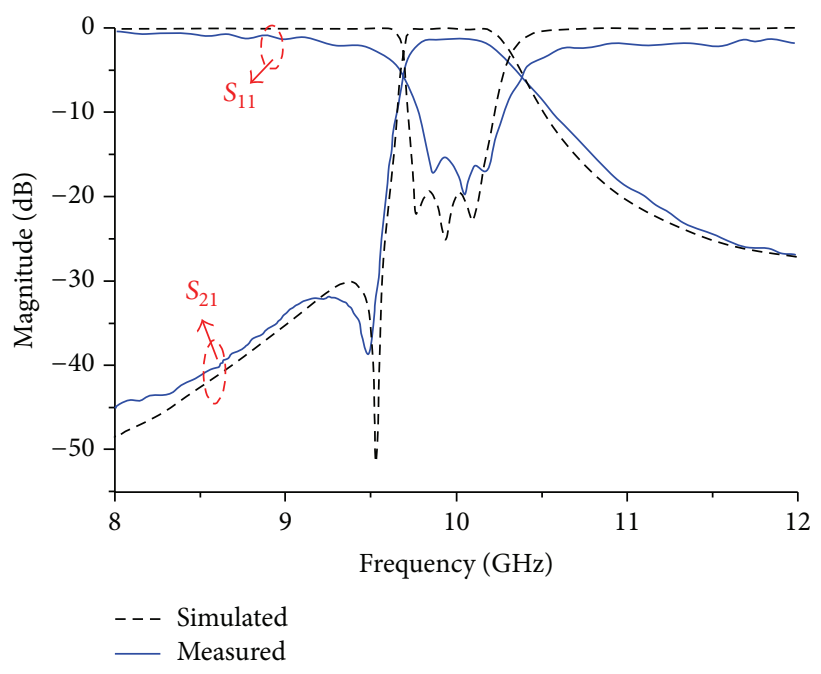

(b)

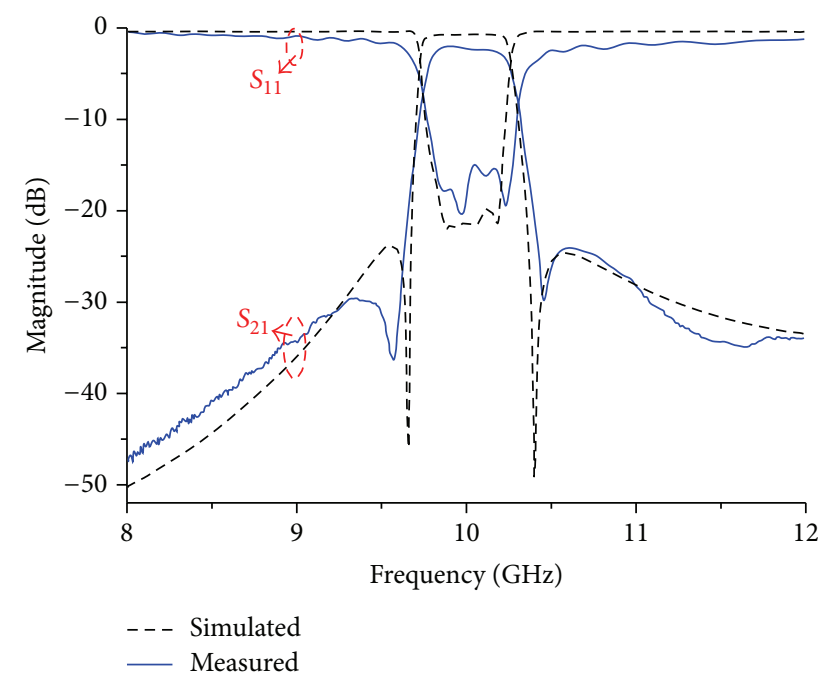

(c)

FIGURE 7: Simulated and measured frequency responses: (a) Filter A, (b) Filter B, and (c) Filter C, respectively.

proposed filters have potential applications for high-density microwave communication.

\section{Acknowledgments}

This work was supported by the National Natural Science Foundation of China (Grants nos. 51172034, 61201001, and 61301052) and the Fundamental Research Funds for the Central Universities of China (Grant no. ZYGX2011J132).

\section{References}

[1] D. Deslandes and K. Wu, "Single-substrate integration technique of planar circuits and waveguide filters," IEEE Transactions on Microwave Theory and Techniques, vol. 51, no. 2, pp. 593-596, 2003.

[2] X.-P. Chen, K. Wu, and D. Drolet, "Substrate integrated waveguide filter with improved stopband performance for satellite ground terminal," IEEE Transactions on Microwave Theory and Techniques, vol. 57, no. 3, pp. 674-683, 2009.

[3] Z. Q. Xu, Y. Shi, C. Y. Xu, and P. Wang, "A novel dual mode substrate integrated waveguide filter with mixed source-load coupling (MSLC)," Progress in Electromagnetics Research, vol. 136, pp. 595-606, 2013.

[4] W. Shen, W.-Y. Yin, and X.-W. Sun, "Miniaturized dual-band substrate integrated waveguide filter with controllable bandwidths," IEEE Microwave and Wireless Components Letters, vol. 21, no. 8, pp. 418-420, 2011.

[5] L.-S. Wu, X.-L. Zhou, W.-Y. Yin, L. Zhou, and J.-F. Mao, "A substrate-integrated evanescent-mode waveguide filter with nonresonating node in low-temperature co-fired ceramic," IEEE Transactions on Microwave Theory and Techniques, vol. 58, no. 10, pp. 2654-2662, 2010.

[6] H. J. Tang, W. Hong, Z. C. Hao, J. X. Chen, and K. Wu, "Optimal design of compact millimetre-wave SIW circular cavity filters," Electronics Letters, vol. 41, no. 19, pp. 1068-1069, 2005. 
[7] Z. Q. Xu, P. Wang, J. X. Liao, and Y. Shi, "Substrate integrated waveguide filter with mixed coupled modified trisections," Electronics Letters, vol. 49, no. 7, pp. 482-483, 2013.

[8] D. de Carlo and S. Tringàli, "Automatic design of circular siw resonators by a hybrid approach based on polynomial fitting and SVRMS," Journal of Electromagnetic Waves and Applications, vol. 24, no. 5-6, pp. 735-744, 2010.

[9] X. P. Chen and K. Wu, "Self-packaged millimeter-wave substrate integrated waveguide filter with asymmetric frequency response," IEEE Transactions on Components, Packaging and Manufacturing Technology, vol. 2, no. 5, pp. 775-782, 2012.

[10] Z. Q. Xu, Y. Shi, P. Wang, J. X. Liao, and X. B. Wei, "Substrate integrated waveguide (SIW) filter with hexagonal resonator," Journal of Electromagnetic Waves and Applications, vol. 26, no. 11-12, pp. 1521-1527, 2012.

[11] Z.-G. Zhang, Y. Fan, Y.-J. Cheng, and Y.-H. Zhang, "A compact multilayer dual-mode substrate integrated circular cavity (SICC) filter for X-band application," Progress in Electromagnetics Research, vol. 122, pp. 453-465, 2012.

[12] W. Jiang, W. Shen, L. Zhou, and W. Y. Yin, "Miniaturized and high-selectivity substrate integrated waveguide (SIW) bandpass filter loaded by complementary split-ring resonators (CSRRs)," Journal of Electromagnetic Waves and Applications, vol. 26, no. 11-12, pp. 1448-1459, 2012.

[13] Z.-G. Zhang, Y. Fan, Y.-J. Cheng, and Y.-H. Zhang, "A novel multilayer dual-mode substrate integrated waveguide complementary filter with circular and elliptic cavities (SICC and SIEC)," Progress in Electromagnetics Research, vol. 127, pp. 173188, 2012. 